\title{
Computerized tomography before the final treatment cycle of neoadjuvant chemotherapy or induction chemotherapy in muscle- invasive urinary bladder cancer, cannot predict pathoanatomical outcomes and does not reflect prognosis-results of a single centre retrospective prognostic study
}

\author{
Haben Mogos ${ }^{1 \#}$, Elisabeth Eriksson ${ }^{2 \#}$, Johan Styrke $^{1 *}$, Amir Sherif ${ }^{\text {*^}}$ \\ ${ }^{1}$ Department of Surgical and Perioperative Sciences, Urology and Andrology, ${ }^{2}$ Department of Radiation Sciences, Diagnostic Radiology, Umea \\ University, Umea, Sweden \\ Contributions: (I) Conception and design: All authors; (II) Administrative support: A Sherif, J Styrke; (III) Provision of study materials or patients: \\ A Sherif, E Eriksson; (IV) Collection and assembly of data: A Sherif, H Mogos, E Eriksson; (V) Data analysis and interpretation: All authors; (VI) \\ Manuscript writing: All authors; (VII) Final approval of manuscript: All authors. \\ \#These authors contributed equally to this study. \\ *These authors contributed equally for the senior authorship. \\ Correspondence to: Dr. Amir Sherif. Department of Surgical and Perioperative Sciences, Urology and Andrology, Umea University, 90185 Umea, \\ Sweden. Email: amir.m.sherif@gmail.com; amir.sherif@umu.se.
}

\begin{abstract}
Background: Evaluating the routine of using control computer tomography (cCT) for determining the response status of muscle-invasive bladder cancer (MIBC) prior to final cycle of neoadjuvant chemotherapy (NAC) or induction chemotherapy (IC), in terms of predicting histopathological pTNM-staging and pathoanatomical responses/non-responses. Secondly, predicting two and three-year overall survival (OS).

Methods: Seventy-seven patients with localized MIBC (cT2-4aN0M0) and 3 patients with minimal nodal dissemination (cN1-2), undergoing NAC or IC and radical cystectomy (RC), the years 2006-2014 at Norrland university hospital in Umeå, Sweden. Baseline pre-cystectomy CTs and cCTs prior to final chemotherapy-cycle, were reviewed and underwent attempted RECIST-criteria categorization, into five response/non-response related subgroups $(n=71)$. The diagnostic accuracy of cCT in comparison with pTNM was assessed using sensitivity, specificity, positive- and negative likelihood ratios. OS for 2 and 3 years was calculated, both in relation to histopathological pTNM-stages in all patients $(n=80)$ and for the patients with cCT-evaluated categories $(n=71)$. Multivariable analysis for OS, was performed in correlation to pTNM-stages firstly, and to radiological staging secondly.

Results: The sensitivity of cCT to predict non-responders according to pTMN was $64 \%$ and specificity $36 \%$. The positive likelihood ratio $=1$ and the negative likelihood ratio $=1$. CT-evaluations couldn't accurately predict pTNM-stages in terms of response/non-response. No statistically significant results were found in correlating cCTs with two and three-year OS.

Conclusions: cCT prior to planned final preoperative chemotherapy-cycle in MIBC patients undergoing NAC or IC, has a poor correlation with pTNM and cannot predict pathoanatomical responses. Prediction of OS based on cCTs is unfeasible.
\end{abstract}

Keywords: Computed X ray tomography; cystectomy interdisciplinary health team; neoadjuvant therapy; urinary bladder neoplasms

Submitted Dec 16, 2019. Accepted for publication May 16, 2020.

doi: $10.21037 /$ tau-19-872

View this article at: http://dx.doi.org/10.21037/tau-19-872

^, ORCID ID: 0000-0002-3675-3050 


\section{Introduction}

In the Western world, urinary bladder cancer (UBC) is the $4^{\text {th }}$ most common malignancy in men and $8^{\text {th }}$ most common in women (1). While most newly diagnosed patients have non-muscle-invasive bladder cancer, urothelial muscle-invasive bladder cancer (MIBC) accounts for approximately $25 \%$ of new cases, with a five-year overall survival (OS) of approximately $50 \%$ in stages cT2-T4 after radical cystectomy (2). MIBC is associated with high risk of regional and distant metastatic spread, the latter with a median survival of 15 months albeit maximum oncological treatment (3). The rationale for Cisplatinbased neoadjuvant combination chemotherapy (NAC) is to eradicate micrometastatic disease at the best point of time, to be followed by radical cystectomy (RC) with regional lymph node dissection $(2,4,5)$.

NAC has been shown to significantly increase OS for a chemo-sensitive sub-group of these patients with an absolute risk reduction (ARR) of $31 \%$ in completely downstaged patients (pT0N0M0) at five years median observation time (6). In a meta-analysis investigating 886 patients from 13 trials, the correlation between complete response (CR i.e., pTONOM0) and improved OS has been reconfirmed (7).

Standard treatment in Sweden, is three, and in some national centers four cycles of NAC, followed by RC, four to five weeks after final chemotherapy cycle (8).

In many centers in Sweden, including Norrland University hospital, Umeå (NUS), there is a routine of performing a control computerized tomography (cCT) prior to the final NAC-cycle. The same routine is used for patients undergoing induction chemotherapy (IC); namely patients with urothelial MIBC and minimal nodal dissemination (cN1-2) yet fit for both preoperative chemotherapy as well as RC. The claimed rationale for the approach of cCT, is to evaluate if the primary tumor is showing response to treatment or to identify regional or general progression of the cancer, comparing with baseline CT images obtained before start of NAC. In case of local progression, the ad hoc recommendation is to proceed quickly to $\mathrm{RC}$ without performing the final cycle of NAC/IC. In cases of very advanced progression or newly discovered disseminated disease-mainly for NAC-patients, the local algorithm is to consider abortion of RC and instead proceed to oncological treatment options. There is one single retrospective study supporting this routine, in which the investigators evaluated 59 patients undergoing
NAC for MIBC. The study concluded that it was possible to evaluate the radiological response rate by pretreatment and post-chemotherapy CT, and that it was feasible to predict both the pathological outcome as well as the postRC survival prognosis (9).

In the Swedish Northern health region (one of six national health regions), all newly diagnosed MIBC-patients are discussed regularly on a weekly basis, at the regional multidisciplinary team (MDT) meetings. For NACpatients and fit patients with minimal nodal disease (pN1-2), the MDT-meetings take place in at least two occasions; primarily after conclusive histopathology following transurethral resection of the bladder tumour (TURb) and baseline CTs have been performed (CT urography and CT Thorax) and later on, following the second of three planned NAC-cycles (in some patients following the third of four planned NAC-cycles), the cCTs are performed and discussed. The standard MDT includes participation of urologists from the whole health region over audio-visual link, oncologists, radiologists, pathologists and specialized nurses. Organized and regular MDT-meetings are considered being highly recommendable for both increasing the usage of NAC and IC, as well as for improving options for diversified treatment selections for all MIBCpatients (10). Apart from the reported single center experience, the lack of solid evidence for the practice of performing a cCT before final NAC cycle, has specifically been addressed by the European Association of Urology and its guidelines-group on Muscle-invasive and Metastatic Bladder Cancer. A variety of imaging modalities (PET, CT, conventional MRI or DCE MRI) are clearly mentioned in their respective incapacity to accurately predict NACresponses $(2,9)$. The primary aim of the study was to analyze the ability of cCT, before the final chemotherapy cycle in mainly NAC patients, to predict histopathological pTNMstaging in terms of response and non-response to NACthat concept considered being an established surrogate marker for prognostication in NAC patients $(6,11)$. Secondary aims were to evaluate the ability of cCT to predict two- and three-year overall survival. We present this article in accordance with the STROBE reporting checklist (available at http://dx.doi.org/10.21037/tau-19-872).

\section{Methods}

Inclusion criteria for this retrospective study, were patients who were diagnosed with urothelial muscle-invasive bladder cancer with or without squamous epithelial differentiation 
Table 1 Baseline variables, comorbidity, staging and histopathological findings

\begin{tabular}{|c|c|c|c|}
\hline Constants & Variables & $\mathrm{n}$ & $\%$ \\
\hline Gender & Male & 60 & 75 \\
\hline & Female & 20 & 25 \\
\hline Median age & \multicolumn{3}{|c|}{68 years (range $44-80$ ) } \\
\hline Comorbidity & CACI-score 2 & 3 & 4 \\
\hline \multirow{7}{*}{$\begin{array}{l}\text { CACI, median } \\
\text { score } 5 \\
\text { (range 2-9) }\end{array}$} & CACl-score 3 & 5 & 6 \\
\hline & CACl-score 4 & 24 & 30 \\
\hline & CACl-score 5 & 29 & 36 \\
\hline & CACl-score 6 & 12 & 15 \\
\hline & CACl-score 7 & 3 & 4 \\
\hline & CACl-score 8 & 3 & 4 \\
\hline & CACl-score 9 & 1 & 1 \\
\hline \multirow{5}{*}{$\begin{array}{l}\text { ASA, median } \\
\text { score } 2 \\
\text { (range 1-4) }\end{array}$} & ASA 1 & 6 & 8 \\
\hline & ASA 2 & 42 & 53 \\
\hline & ASA 3 & 30 & 38 \\
\hline & ASA 4 & 1 & 1 \\
\hline & ASA unknown & 1 & 1 \\
\hline \multirow[t]{8}{*}{ Clinical TNM } & cT2 & 31 & 39 \\
\hline & сT3 & 38 & 47 \\
\hline & cT4a & 11 & 14 \\
\hline & cNO & 77 & 96 \\
\hline & cN1 & 2 & 2 \\
\hline & cN2 & 1 & 1 \\
\hline & cMO & 80 & 100 \\
\hline & cM1 & 0 & 0 \\
\hline \multirow[t]{13}{*}{ Pathological TNM } & рTO & 37 & 46 \\
\hline & pTa & 2 & 2.5 \\
\hline & pTis & 4 & 5 \\
\hline & pT1 & 2 & 2.5 \\
\hline & pT2 & 19 & 24 \\
\hline & pT3 & 7 & 9 \\
\hline & pT4 & 9 & 11 \\
\hline & pNo & 64 & 80 \\
\hline & $\mathrm{pN} 1$ & 5 & 6 \\
\hline & pN2 & 3 & 4 \\
\hline & $\mathrm{pNX}$ & 8 & 10 \\
\hline & pM0 & 100 & 100 \\
\hline & pM1 & 0 & 0 \\
\hline
\end{tabular}

Table 1 (Continued)
Table 1 (Continued)

\begin{tabular}{lcll}
\hline Constants & Variables & $\mathrm{n}$ & $\%$ \\
\hline Histopathology & Urothelial & 67 & 84 \\
& Urothelial + & 13 & 16 \\
& $\begin{array}{l}\text { Squamous } \\
\text { differentiation }\end{array}$ & & \\
\hline
\end{tabular}

The 60 men and 20 women had a median age of 68 years and many had significant comorbidity $(50 \%$ being classified as ASA $\geq 3$ ). The clinical T-stage was cT3 or cT4 in 49 cases but in the post cystectomy specimens only 16 cases of pT3 or pT4 was found. Ten patients had positive lymph nodes, but no patient had been diagnosed with M1-disease at the time of post cystectomy histopathological review. $\mathrm{CACl}$, Charlson age-adjusted Comorbidity Index. ASA, American Society of Anesthesiologists Physical Status-system.

from the internal department-list of all patients who had undergone NAC and RC between the years 2006-2014 at a tertiary referral center - Norrland university hospital (NUS) in Umeå, Sweden. Seventy-seven NAC-patients with localized urothelial MIBC (cT2-4aN0M0) were identified, plus three patients with minimal nodal disease (cN1-2). The study database included patient data from all available patient records, as well as radiological and perioperative data from necessary documentation systems used at NUS. The included clinical data were; age, gender, clinical and pathological TNM staging, pre-RC CACI (Charlson ageadjusted Comorbidity Index), pre-RC ASA (Table 1) and specifications on NAC treatment (type, amount of cycles) (Table 2). For cTNM-staging, patients with uni- or bilateral hydronephrosis were classified as cT3. Baseline CTs (CT urography and CT thorax) were performed in conjunction with diagnostic TURb, to determine $\mathrm{cN}$-status and $\mathrm{cM}$ status. Patients with suspicious $\mathrm{cM}+$ status, were not included in the study. For describing the whole RC-series of MIBC-patients receiving pre-RC chemotherapy the given years at our center, we also decided to include the three patients who received induction chemotherapy (two patients with $\mathrm{cN} 1$-disease and one staged preoperatively as $\mathrm{cN} 2$ ). Induction chemotherapy (IC) is a treatment strategy which is not defined as bona fide NAC-treatment, but for the completeness of the series these patients were also evaluated (2). For pTNM-staging post-RC we set following designations; pT0N0M0 for complete response (CR), pTaN0M0, pTisN0M0 or pT1N0M0 for partial response (PR), pT2-T4aN0M0 for stable disease (SD), pN+ and/ or $\mathrm{pM}+\mathrm{pT} 4 \mathrm{~b}$ and/or tumour positive resection margin for progressive disease (PD). 


\section{RECIST criteria}

The radiologist in the research group (EE), reviewed all patients base line CTs and the CTs performed prior to final chemotherapy cycle, with the intentions to classify the evaluations according to RECIST-criteria (Response Evaluation Criteria In Solid Tumors) into following categories; complete response (CR), stable disease (SD) or progressive disease (PD). For not mixing these evaluations

Table 2 Type of neoadjuvant chemotherapy and number of fulfilled cycles

\begin{tabular}{lcc}
\hline NAC variables & $\mathrm{n}$ & $\%$ \\
\hline NAC Treatment & 75 & 94 \\
MVAC \& HD-MVAC & 5 & 6 \\
Cisplatin-Gemzar & & \\
Number of NAC-cycles & 1 & 1 \\
One cycle & 9 & 11 \\
Two cycles & 46 & 58 \\
Three cycles & 23 & 29 \\
Four cycles & 1 & 1 \\
Five cycles & & \\
\hline
\end{tabular}

MVAC or HD-MVAC was the dominating chemotherapy regimen. Twelve patients could not continue to three or more NAC-cycles because of significant side effects or suspected non-response. NAC, neoadjuvant chemotherapy; MVAC, methotrexate, vinblastine, adriamycin and cisplatin; HD-MVAC, high-dose MVAC. with the similar set of response designations for postRC histopathological evaluations, we designated CTevaluations of complete response with CR-CT, stable disease with SD-CT, and progressive disease with PDCT. Further our radiologist had to expand the number of radiological groups, to also include two new designations; mixed response (MIXRESP-CT), and difficult to interpret (DIFINTRP-CT) (Table 3).

\section{Statistics}

Standard descriptive statistics were used to display the findings in the results-section. First, we evaluated the results of the cCT scans in relation to the pathoanatomical outcomes post-RC. The index tests which were used, were the results of the cCTs performed prior to the final chemotherapy cycle. As the reference test for pathoanatomical outcomes, pTNM staging was used. The analysis of the primary outcome measurement included the 56 patients that could be dichotomized into either complete response (CR-CT) or no response; i.e., stable disease plus progressive disease (SD-CT + PD-CT) who had undergone complete assessments of both pathology post-RC as well as computed tomography. The main cohort of 80 patients was then analyzed in terms of OS at two- and further at three-year median time of observation, based on the different subgroups of pTNM. From the 80 patients, the 71 patients with complete radiological data, also including those with mixed response (MIXRESP-CT) and those who had scans difficult to interpret (DIFINTRP-CT) were then

Table 3 The findings of the follow-up CT-scan in comparison to the baseline CT-scan

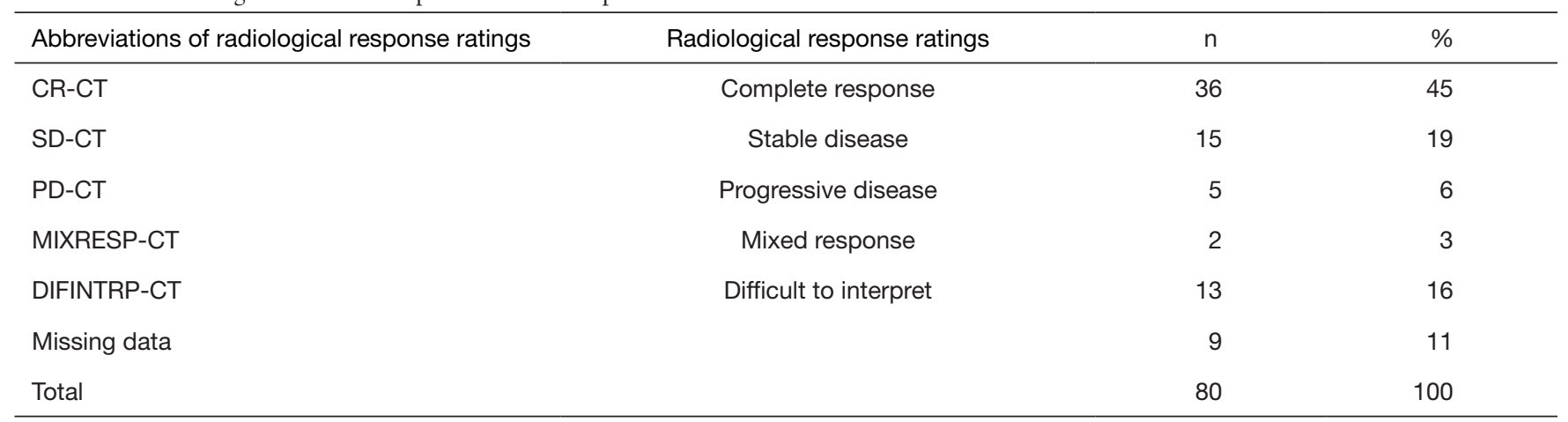

Almost half of the patients showed radiological signs of regression according to the RECIST criteria. Notably, 15 patients had mixed response or images that were difficult to interpret thus leaving a group of 56 patients for dichotomized analysis of diagnostic ability. CT, computed tomography; CR-CT, complete response/regression computed tomography; SD-CT, computed tomography stable disease; PD$\mathrm{CT}$, computed tomography progressive disease; MIXRESP-CT, computed tomography mixed response; DIFINTRP-CT, computed tomography difficult to interpret. 


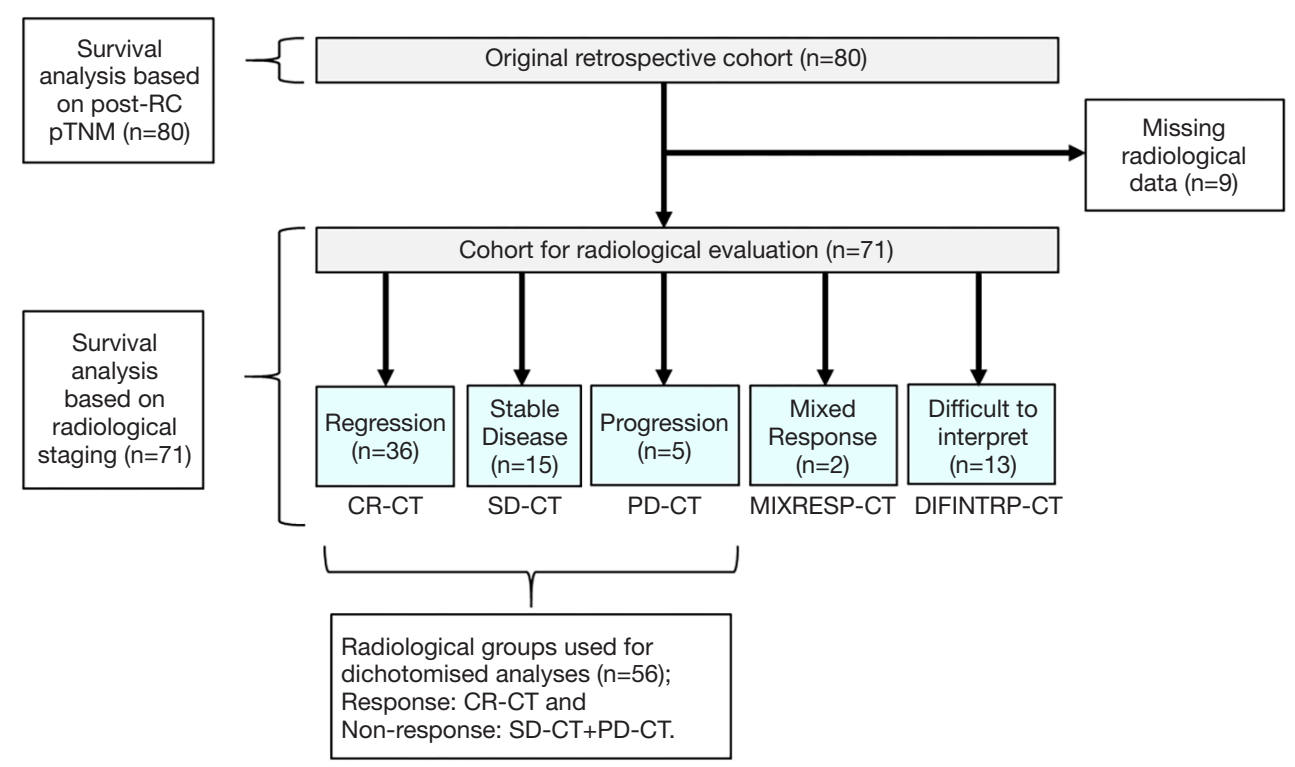

Figure 1 Study flow chart of the study population showing the results of the radiological assessment and the different groups used for outcome analysis. The flow chart of the cohort of 60 men and 20 women included in the study shows the proportion of patients categorized into different radiological groups. Almost half of the patients showed radiological signs of regression according to the RECIST criteria. Notably, 15 patients had mixed response or images that were difficult to interpret thus leaving a group of 56 patients for dichotomized analysis of diagnostic ability. RC, radical cystectomy; pTNM, histopathological staging; CT, computed tomography; R-CT, computed tomography regression; SD-CT, computed tomography stable disease; PD-CT, computed tomography progressive disease; MIXRESP-CT, computed tomography mixed response; DIFINTRP-CT, computed tomography difficult to interpret.

analyzed for OS at two- and for three-years median time of observation, based on the different subgroups of radiological staging (Figure 1). The same analysis was conducted using the pTNM-groups. A logistic regression was performed using the pTNM-groups and cCT-groups to analyze the two and three-year OS, since the outcome death, prior to two or three years, were expressed as binary events 1 or 0 . The analyses were adjusted for age, gender and comorbidity (CACI). The data was analysed with IBM SPSS statistics 24, where we set a $\mathrm{P}$ value $<0.05$ as the indicator of statistical significance.

\section{Results}

The cohort had a female-to-male ratio of $1: 3$, a median age of 68 at RC, a median comorbidity score (CACI) of 5 and a median ASA score of 2. The pathological TNM-staging revealed that $46 \%$ of the patients were completely down staged to pT0, $10 \%$ had positive lymph nodes but none had been diagnosed with distant metastases (Table 1). Most patients had received MVAC or HD-MVAC (94\%) and $87 \%$ had undergone three or four cycles of preoperative chemotherapy (NAC or IC) (Table 2). The results of the CT-analysis are shown in Table 3. Complete response (CRCT) was found in $45 \%$ of the patients while $25 \%$ of the patients were considered to be non-responders (stable disease; SD-CT plus progressive disease PD-CT). Almost $20 \%$ of the patients had mixed response (MIXRESP-CT) or scans that were difficult to interpret (DIFINTRP-CT). The 56 patients who had undergone complete assessments of both pathology post-RC as well as cCT, with results that could be dichotomized into either complete response (CR$\mathrm{CT}$ ) or no response (SD-CT+PD-CT), were evaluated in comparison with the corresponding pTNM-staging of the post-RC specimens. The sensitivity of CT to predict non-responders according to pTNM was 64\% (95\% CI: $44-81 \%$ ), the specificity was $36 \%$ (95\% CI: $19-56 \%$ ) the positive likelihood ratio was 1 (95\% CI: $0.7-1.5)$ and the negative likelihood ratio was 1 (95\% CI: $0.5-2)$. The predictive results were evenly distributed (fifty/fifty) and thus of no avail for accurate prediction (Table 4). When evaluating the 71 patient that also included those with MIXRESP-CT and those who were deemed DIFINTRPCT we found that only $14 / 36$ patients (39\%) with CT- 
Table 4 Cross-tabulation of the 56 patients who were assessed to have response or no response on computed tomography in comparison with the corresponding pathological findings

\begin{tabular}{lccc}
\hline & p-response (CR+PR) & p-no response (SD+PD) & Total \\
\hline CR-CT & 18 & 18 & 36 \\
CT-No response (SD-CT+PD-CT) & 10 & 10 & 20 \\
Total & 28 & 28 & 56 \\
\hline
\end{tabular}

The table shows that half of the patients with an assessed complete response on CT-imaging in fact had no pathological response at all and that half of the patients assessed to have no response on CT-imaging had complete or partial response according to the results of the pathological analysis. p-PD, pathological progressive disease; CR-CT, complete response/regression computed tomography; SD-CT, computed tomography stable disease; PD-CT, computed tomography progressive disease; MIXRESP-CT, computed tomography mixed response; DIFINTRP-CT, computed tomography difficult to interpret.

Table 5 Cross-tabulation of the 71 patients who had complete assessments for pathological findings and computed tomography findings

\begin{tabular}{lccccc}
\hline & CR & PR & SD & PD & Total \\
\hline CR-CT & 14 & 4 & 12 & 6 & 0 \\
SD-CT & 6 & 0 & 9 & 15 \\
PD-CT & 4 & 0 & 0 & 5 \\
MIXRESP-CT & 0 & 0 & 0 & 2 & 1 \\
DIFINTRP-CT & 7 & 4 & 1 & 13 \\
Total & 31 & 8 & 22 & 10
\end{tabular}

The table shows a poor correlation between CT- and pathology-findings; the 36 patients with an assessed complete response on CTimaging had a distribution of pathological findings all across the spectrum. For example, six of the 10 patients with progressive disease had a CT-scan signaling complete response. Of the 13 patients with images difficult to interpret, eleven had complete or partial response. $\mathrm{CR}$, pathological complete response; PR, pathological partial response; SD, pathological stable disease; PD, pathological progressive disease; CR-CT, complete response/regression computed tomography; SD-CT, computed tomography stable disease; PD-CT, computed tomography progressive disease; MIXRESP-CT, computed tomography mixed response; DIFINTRP-CT, computed tomography difficult to interpret.

predicted regression had complete response (CR) and as many as six of 36 patients (17\%) had progressed (PD). Notably four of five patients $(80 \%)$, with computer tomography evaluations of PD (PD-CT), actually were CR; thus, only one of five $(20 \%)$ had been correctly predicted with progressive disease (Table 5).

The odds ratio for two-year OS was calculated with pathological CR (pTONOM0) as reference. A statistically significant increase in death, was seen in the pathological $\mathrm{SD}$ and pathological PD patient groups compared to CR patients $(\mathrm{P}=0.003$ and $\mathrm{P}=0.005$ respectively). The same correlation could not be seen when analyzing survival for $\mathrm{PR}$ patients to $\mathrm{CR}(\mathrm{P}=0.86)$. The patients with progressive disease had 8.96 timed higher odds of dying within two years after cystectomy when compared to the patients with complete response (Table 6). Similar results were found when analyzing three-year survival in the same groups with a statistically significant increase in death for patients in the pathological SD and $\mathrm{PD}$ patient groups compared to $\mathrm{CR}$ ( $\mathrm{P}=0.03$ and resp. $\mathrm{P}=0.009$ ) (Table 7).

No statistically significant results were observed when analyzing two-year OS in comparing the radiological responses of the different groups (CR-CT, PD-CT, MIXRESP-CT and resp. DIFINTRP-CT) with the group of patients that radiologically were deemed to have a stable disease (SD-CT), in comparison with their respective baseline CT (Table 8). Analysis of the three-year OS in the same groups gave similar results as the two-year OS analysis (Table 9). Thus, the radiological response measured in CTevaluations did not correlate to two- or three-year survival with any statistical significance.

We also analyzed two and three-year OS, only considering the patients with complete response (CR-CT) compared to the group with radiological stable disease (SD- 
Table 6 Odds ratios of death within two years after radical cystectomy depending on pathological staging of the cystectomy specimens adjusted for age and comorbidity

\begin{tabular}{lllll}
\hline & B & OR & 95\% Cl & P value \\
\hline Age at RC & 0.07 & 1.07 & $0.97-1.19$ & 0.19 \\
CACl & -0.35 & 0.70 & $0.38-1.32$ & 0.27 \\
PR compared with CR & 0.22 & 1.24 & $0.12-13.40$ & 0.86 \\
SD compared with CR & 2.04 & 7.66 & $2.03-28.98$ & $0.003^{*}$ \\
PD compared with CR & 2.19 & 8.96 & $1.93-41.56$ & $0.005^{\star *}$ \\
Constant & -4.96 & 0.007 & - & 0.07 \\
\hline
\end{tabular}

The logistic regression analyzing death within two years of cystectomy depending on pathological staging showed two significant results:

*, the patients with pathological stable disease had 7.66 times higher odds of dying within two years after cystectomy when compared to the patients with pathological complete response. ${ }^{* *}$, the patients with pathological progressive disease had 8.96 timed higher odds of dying within two years after cystectomy when compared to the patients with pathological complete response. B, unstandardized regression coefficient; OR, odds ratio; $\mathrm{Cl}$, confidence interval; RC, radical cystectomy; $\mathrm{CACl}$, Charlson age-adjusted Comorbidity Index; $\mathrm{PR}$, pathological partial response; $\mathrm{CR}$, complete response; SD, stable disease; PD, progressive disease.

Table 7 Odds ratios of death within three years after radical cystectomy depending on pathological staging of the cystectomy specimens adjusted for age and comorbidity

\begin{tabular}{lcccc}
\hline & B & OR & 95\% Cl & $0.92-1.09$ \\
\hline Age at RC & -0.003 & 1.00 & $0.67-1.79$ & 0.95 \\
CACI & 0.09 & 1.09 & 0.73 \\
PR compared with CR & -0.85 & 0.43 & $0.05-4.03$ & 0.46 \\
SD compared with CR & 1.25 & 3.50 & $1.15-10.69$ & $0.03^{\star}$ \\
PD compared with CR & 1.89 & 6.63 & $1.62-27.13$ & $0.009^{\star \star}$ \\
Constant & -1.31 & 0.27 & - & 0.57 \\
\hline
\end{tabular}

The logistic regression analyzing death within three years of cystectomy depending on pathological staging showed two significant results: *, the patients with pathological stable disease had 3.5 times higher odds of dying within two years after cystectomy when compared to the patients with pathological complete response. ${ }^{* *}$, the patients with pathological progressive disease had 6.63 timed higher odds of dying within two years after cystectomy when compared to the patients with pathological complete response. B, unstandardized regression coefficient; OR, odds ratio; $\mathrm{Cl}$, confidence interval; RC, radical cystectomy; CACI, Charlson age-adjusted Comorbidity Index; PR, pathological partial response; CR, pathological complete response; SD, pathological stable disease; PD, pathological progressive disease.

CT), without identifying any statistically significant results (Tables 10,11).

\section{Discussion/Conclusions}

The introduction of NAC plus $\mathrm{RC}$, as a recommendation of treatment for all medically fit patients with urothelial MIBC, is based on a set of well performed randomized prospective trials displaying survival benefits (OS) ranging from $5-8 \%$ at five years of median observation time $(2,4,5)$. Yet, as it is well known in analyzing both the mentioned trials as well as several register-based investigations, only $25-40 \%$ of treated patients show CR or PR-these pathoanatomical outcomes in their respective capacities of being surrogate markers of treatment efficacy in terms of longtime survival benefits $(6,11,12)$. Thus, most of the patients will either have SD or PD even after three cycles of NAC and will consequently have received treatment leaving no impact on survival improvement. The delays for radical treatment (mainly RC) in that large subset of patients, are mainly ranging from 2.5 to four months, depending on specific chemotherapy regimen and local tradition-a delay that might hypothetically also have negative impact on the survival projections. Yet, there are yet no studies showing 
Table 8 Odds ratios of death within two years after radical cystectomy depending on computed tomography staging after the second NAC-cycle adjusted for age and comorbidity

\begin{tabular}{lcccc}
\hline & B & OR & 95\% Cl & $0.99-1.25$ \\
\hline Age at RC & 0.11 & 1.11 & $0.23-1.08$ & 0.07 \\
CACl & -0.71 & 0.49 & $0.18-2.86$ \\
CR-CT compared with SD-CT & -0.32 & 0.72 & $0.16-13.70$ \\
PD-CT compared with SD-CT & 0.40 & 1.49 & $0.112-50.40$ \\
MIXRESP-CT compared with SD-CT & 0.87 & 2.38 & $0.04-2.02$ & 0.58 \\
DIFINTRP-CT compared with SD-CT & -1.21 & 0.30 & - & 0.22 \\
Constant & -4.62 & 0.01 & 0.14 \\
\hline
\end{tabular}

The logistic regression analyzing death within two years of cystectomy depending on cCT-staging showed no significant results; CTstaging could not predict death after two years. NAC: neoadjuvant chemotherapy; B, unstandardized regression coefficient; OR: odds ratio; $\mathrm{Cl}$, confidence interval; $\mathrm{RC}$, radical cystectomy; $\mathrm{CACl}$, Charlson age-adjusted Comorbidity Index; CR-CT, complete response/ regression computed tomography; SD-CT, computed tomography stable disease; PD-CT, computed tomography progressive disease; MIXRESP-CT, computed tomography mixed response; DIFINTRP-CT, computed tomography difficult to interpret.

Table 9 Odds ratios of death within three years after radical cystectomy depending on computed tomography staging after the second NAC-cycle adjusted for age and comorbidity

\begin{tabular}{lcccc}
\hline & B & OR & 95\% Cl & $0.93-1.11$ \\
\hline Age at RC & 0.02 & 1.02 & $0.57-1.57$ & 0.84 \\
CACI & -0.05 & 0.95 & $0.22-2.68$ \\
CR-CT compared with SD-CT & -0.27 & 0.77 & $0.54-72.03$ \\
PD-CT compared with SD-CT & 1.83 & 6.25 & $0.08-32.07$ \\
MIXRESP-CT compared with SD-CT & 0.47 & 1.60 & $0.13-3.13$ & 0.14 \\
DIFINTRP-CT compared with SD-CT & -0.44 & 0.65 & 0.76 \\
Constant & -1.27 & 0.28 & 0.59 \\
\hline
\end{tabular}

The logistic regression analyzing death within three years of cystectomy depending on CCT-staging showed no significant results; CTstaging could not predict death after three years. NAC, neoadjuvant chemotherapy; B, unstandardized regression coefficient; OR, odds ratio; $\mathrm{Cl}$, confidence interval; $\mathrm{RC}$, radical cystectomy; $\mathrm{CACl}$, Charlson age-adjusted Comorbidity Index; CR-CT, complete response/ regression computed tomography; SD-CT, computed tomography stable disease; PD-CT, computed tomography progressive disease; MIXRESP-CT, computed tomography mixed response; DIFINTRP-CT, computed tomography difficult to interpret.

Table 10 Odds ratios of death within two years after radical cystectomy depending on the computed tomography staging groups regression or stable disease after the second NAC-cycle adjusted for age and comorbidity

\begin{tabular}{|c|c|c|c|c|}
\hline & $\mathrm{B}$ & OR & $95 \% \mathrm{Cl}$ & $P$ value \\
\hline $\mathrm{CACl}$ & -0.96 & 0.38 & $0.13-1.13$ & 0.08 \\
\hline CR-CT compared with SD-CT & -0.45 & 0.64 & $0.16-2.58$ & 0.53 \\
\hline Constant & -2.16 & 0.12 & - & 0.54 \\
\hline
\end{tabular}

The logistic regression analyzing death within two years of cystectomy depending on the cCT-staging-groups regression or stable disease showed no significant results; CT-staging could not predict death after two years. NAC, neoadjuvant chemotherapy; B, unstandardized regression coefficient; OR, odds ratio; $\mathrm{Cl}$, confidence interval; $\mathrm{RC}$, radical cystectomy; $\mathrm{CACl}$, Charlson age-adjusted Comorbidity Index; $\mathrm{CR}-\mathrm{CT}$, complete response/regression computed tomography; SD-CT, computed tomography stable disease. 
Table 11 Odds ratios of death within three years after radical cystectomy depending on the computed tomography staging groups regression or stable disease after the second NAC-cycle adjusted for age and comorbidity

\begin{tabular}{lcccc}
\hline & B & OR & $95 \%$ Cl & P value \\
\hline Age at RC & 0.004 & 1.00 & $0.90-1.10$ & 0.94 \\
CACl & -0.10 & 0.90 & $0.51-1.59$ & 0.72 \\
CR-CT compared with SD-CT & -0.31 & 0.73 & $0.21-2.57$ & 0.63 \\
Constant & 0.37 & 1.45 & - & 0.9 \\
\hline
\end{tabular}

The logistic regression analyzing death within three years of cystectomy depending on cCT-staging-groups regression or stable disease showed no significant results; CT-staging could not predict death after three years. NAC, neoadjuvant chemotherapy; B, unstandardized regression coefficient; OR, odds ratio; $\mathrm{Cl}$, confidence interval; $\mathrm{RC}$, radical cystectomy; $\mathrm{CACl}$, Charlson age-adjusted Comorbidity Index; CR-CT, complete response/regression computed tomography; SD-CT, computed tomography stable disease.

any negative survival impact, due specifically to the delays of NAC treatment. Extended lead times until RC as a negative factor for final pTNM-stages and with negative impact on long term survival, has only been evaluated in NAC-naïve MIBC-patients (i.e., undergoing RC only) (13-15). Further, until now there are no randomized prospective trials for MIBC-patients, evaluating IC plus RC versus chemonaïve treatment (RC only), in terms of survival benefits or pathoanatomical outcomes.

Our study was made to evaluate a routine using a control CT to determine if the patient is responding to neoadjuvant chemotherapy in urothelial MIBC or not. If deemed nonresponsive with radiological signs of progression of cancer, the last cycle of chemotherapy would not be administered and the patient's planned RC would be performed as quickly as possible, alternatively that the patient would be offered a non-surgical oncological option. We compared the outcomes and survival results of the surrogate markers of downstaging in the final cystectomy specimens (urinary bladder tumor with excised regional lymph nodes), to a parallel survival analysis guided by the results of resp. CTscans performed prior to final cycle of NAC. As expected, our study displayed an improved overall survival when having a completely histopathologically downstaged cancer (pTONOM0) at time of cystectomy versus still having a muscle invasive or an upstaged cancer in the main cohort of patients $(n=80)$. Further, we showed that $\mathrm{cCT}$ had a poor correlation with the post-RC pTNM and could not predict OS. In our cohort of CT-evaluated patients $(n=71)$, the patients were placed in groups according to the assessment of their radiological response after their cCT, the diversity of radiologic assessment-groups we had to include, shows an uncertainty using CT as a control method. Instead of only having three groups; regression
(CR-CT), stable disease (SD-CT), and progression (PD$\mathrm{CT}$ ), our radiologist had to expand the number of groups to also include mixed response (MIXRESP-CT), and difficult to interpret (DIFINTRP-CT). The lack of predictive value for the control CT in relation to pathoanatomical response and non-response parameters in the post-RC specimens, was obvious. One major argument for using the control CT, is that it at least can inform the concerned clinicians about progressing patients (PD) and that the question of stable disease or not, is to be considered of less importance.

Yet, $80 \%$ with computer tomography evaluations of PD (PD-CT) had actually CR and only $20 \%$ had been correctly predicted with progressive disease (Table 5) this deeming the control CT in terms of PD-prediction, rather useless. Thus, our findings further strengthen the mentioned negative recommendation from the EAUGuidelines group, regarding the utilization of control CT in clinical practice (2).

Further, we found, when using cCT in routine manner, that there was lack of statistically significant results when comparing OS to CT results. We conclude that the cCT before final NAC cycle, did not contribute to any prognostic information, in our analysis. Yet the study was limited due to its retrospective nature and due to the moderate size of the cohort. A larger cohort would make it possible to adjust results with additional confounders. With a larger cohort, it would also be of interest to separately analyze true NACpatients separately from patients who had undergone IC. Yet, there are at present no described radiological standards for that kind of comparative evaluations. The study covers $100 \%$ of all cystectomized patients fitting to the inclusion criteria at a high output urological department during a given time-period, and in contrast to the Swedish national cystectomy register, we have substantial amounts of detailed 
data on every included patient plus that we have validated all data; patient by patient (8). Larger retrospective trials and prospective trials are warranted.

In conclusion, control CT prior to the planned final preoperative chemotherapy-cycle in MIBC patients undergoing $\mathrm{NAC}$ or induction chemotherapy has a poor correlation with post-RC pTNM and cannot predict overall survival.

\section{Acknowledgments}

Funding: This work was supported by the Swedish Research Council funding for clinical research in medicine (ALF) in Västerbotten, VLL, Sweden and the Cancer Research Foundation in Norrland, Umeå, Sweden. We also thank assoc. Professor Johan Svensson, Umeå School of Business, Economics and Statistics (USBE), Umeå university, Sweden, for valuable support in analysis of the statistics.

\section{Footnote}

Reporting Checklist: The authors have completed the STROBE reporting checklist. Available at http://dx. doi. org/10. 21037/tau-19-872

Data Sharing Statement: Available at http://dx. doi. org/10.21037/tau-19-872

Conflict of Interest: All authors have completed the ICMJE uniform disclosure form (available at http://dx. doi. org/10. 21037/tau-19-872). The authors have no conflicts of interest to declare. None of the authors have at any time received payment or services from a third party for any aspect of the submitted work. None of the authors have any patents; planned, pending or issued, broadly, relevant to the work. The four authors have no other relationships or activities that readers could perceive to have influenced or have given the appearance of potentially influencing the contents in article.

Ethical Statement: The authors are accountable for all aspects of the work in ensuring that questions related to the accuracy or integrity of any part of the work are appropriately investigated and resolved. The study was approved by the Regional Ethics Board in Umeå: EPNUmeå, dnr; 2013/463-31M with the amendment dnr; 2016/403-32. The study conforms to the provisions of the Declaration of Helsinki (as revised in Fortaleza, Brazil,
October 2013). The Regional Ethics Board had specifically decided that informed consent from the participants was to be considered redundant, especially due to the high mortality in MIBC as well as due to the retrospective nature of the study.

Open Access Statement: This is an Open Access article distributed in accordance with the Creative Commons Attribution-NonCommercial-NoDerivs 4.0 International License (CC BY-NC-ND 4.0), which permits the noncommercial replication and distribution of the article with the strict proviso that no changes or edits are made and the original work is properly cited (including links to both the formal publication through the relevant DOI and the license). See: https://creativecommons.org/licenses/by-nc-nd/4.0/.

\section{References}

1. Kirkali Z, Chan T, Manoharan, et al. Bladder cancer: epidemiology, staging and grading, and diagnosis. Urology 2005;66:4-34.

2. Witjes JA, Compérat E, Cowan NC, et al. Updated 2016 EAU Guidelines on Muscle-invasive and Metastatic Bladder Cancer. Eur Urol 2017;71:462-75.

3. von der Maase H, Hansen SW, Roberts JT, et al. Gemcitabine and cisplatin versus methotrexate, vinblastine, doxorubicin, and cisplatin in advanced or metastatic bladder cancer: results of a large, randomized, multinational, multicenter, phase III study. J Clin Oncol 2000;18:3068-77.

4. Sherif A, Holmberg L, Rintala E, et al. Neoadjuvant cisplatinum based combination chemotherapy in patients with invasive bladder cancer: a combined analysis of two Nordic studies. Eur Urol 2004;45:297-303.

5. Advanced Bladder Cancer (ABC) Meta-analysis Collaboration. Neoadjuvant chemotherapy in invasive bladder cancer: update of a systematic review and metaanalysis of individual patient data advanced bladder cancer (ABC) meta-analysis collaboration. Eur Urol 2005;48:2025; discussion 205-6.

6. Rosenblatt R, Sherif A, Rintala E, et al. Pathologic downstaging is a surrogate marker for efficacy and increased survival following neoadjuvant chemotherapy and radical cystectomy for muscle-invasive urothelial bladder cancer. Eur Urol 2012;61:1229-38.

7. Petrelli F, Coinu A, Cabiddu M, et al. Correlation of pathologic complete response with survival after neoadjuvant chemotherapy in bladder cancer treated with 
cystectomy: a meta-analysis. Eur Urol 2014;65:350-7.

8. Jerlström T, Chen R, Liedberg F, et al. No increased risk of short-term complications after radical cystectomy for muscle-invasive bladder cancer among patients treated with preoperative chemotherapy: a nation-wide registerbased study. World J Urol 2020;38:381-8.

9. Fukui T, Matsui Y, Umeoka S, et al. Predictive value of radiological response rate for pathological response to neoadjuvant chemotherapy and post-cystectomy survival of bladder urothelial cancer. Jpn J Clin Oncol 2016;46:560-7.

10. Sherif A. The long perspective in emergence of neoadjuvant chemotherapy for bladder cancer in Ontario, Canada-space for improvement with regular and organized multidisciplinary team meetings. Transl Androl Urol 2018;7:508-10.

11. Lavery HJ, Stensland KD, Niegisch G, et al. Pathological T0 following radical cystectomy with or without neoadjuvant chemotherapy: a useful surrogate. J Urol 2014;191:898-906.

Cite this article as: Mogos H, Eriksson E, Styrke J, Sherif A. Computerized tomography before the final treatment cycle of neoadjuvant chemotherapy or induction chemotherapy in muscle-invasive urinary bladder cancer, cannot predict pathoanatomical outcomes and does not reflect prognosisresults of a single centre retrospective prognostic study. Transl Androl Urol 2020;9(3):1062-1072. doi:10.21037/tau-19-872
12. Zargar H, Zargar-Shoshtari K, Lotan Y, et al. Final Pathological Stage after Neoadjuvant Chemotherapy and Radical Cystectomy for Bladder Cancer-Does pT0 Predict Better Survival than p'Ta/Tis/T1? J Urol 2016;195:886-93.

13. Sánchez-Ortiz RF, Huang WC, Mick R, et al. An interval longer than 12 weeks between the diagnosis of muscle invasion and cystectomy is associated with worse outcome in bladder carcinoma. J Urol 2003;169:110-5; discussion 115 .

14. Chang SS, Hassan JM, Cookson MS, et al. Delaying radical cystectomy for muscle invasive bladder cancer results in worse pathological stage. J Urol 2003;170:1085-7.

15. May M, Nitzke T, Helke C, et al.Significance of the time period between diagnosis of muscle invasion and radical cystectomy with regard to the prognosis of transitional cell carcinoma of the urothelium in the bladder. Scand J Urol Nephrol 2004;38:231-5. 